\title{
Maxwell's Dynamical Philosophy: An Early Solution to the Problem of a Discrepancy between Particles and Waves
}

\author{
Salvo D'Agostino \\ University of Roma La Sapienza, Rome, Italy \\ Email: saldagostino21@gmail.com
}

Received 2 May 2014; revised 5 June 2014; accepted 27 June 2014

Copyright (C) 2014 by author and Scientific Research Publishing Inc. This work is licensed under the Creative Commons Attribution International License (CC BY). http://creativecommons.org/licenses/by/4.0/

(c) (i) Open Access

\section{Abstract}

J. C. Maxwell contributed to an important philosophy of science, the so-called Dynamical Philosophy and Dynamical Approach to his theories. He meant it by a Dynamical Approach a theory of motions of the moving particle that could dispense with the detailed mechanism of motion. In order to develop this type of approach, he applied a modified form of Lagrange's equations in his 1865 essay "A Dynamical Theory of the Electromagnetic Field" and in his master opus "A Treatise on Electricity and Magnetism". But an attentive historical research has also realized that he used an analogous approach in his contribution to a kinetic theory of gases, the well known statistical law of velocities.

\section{Keywords}

Particles and Waves, J. C. Maxwell

\section{Introduction}

The outstanding contributions of J. C. he Maxwell (1831-1879) to a field theory of electromagnetism and to a kinetic theory of gas have been extensively studied in recent years. However, it is scarcely known that Maxwell also contributed to an important philosophy of science, the so-called Dynamical Philosophy and Dynamical Approach to his theories. This paper deals with a study of J. C. Maxwell's Dynamical Approach to his Field theory of Electricity and Magnetism and to his Statistical Gas theory James Clerk Maxwell (Edinburg 1831-Cambridge 1879), was one of the most outstanding personalities of the British School of Dynamical Philosophy.

His initial twin sources of inspiration came by a powerful dynamical conception of nature, that of Faraday, and by the channel of William Thomson's contributions. Faraday and Thomson insisted on the primary impor- 
tance of the dynamical aspect of phenomena and Thomson regarded molecules as vortexes occurring in an essentially continuous medium, which transmitted forces through space. In 1865 Maxwell published a Memoir on the Royal Society Transactions, "A Dynamical Theory of the Electromagnetic Field", in which he referred to Lagranges' Mechanique Analitique as a source for his equations of the electromagnetic field. In his 1973 master opus A Treatise on Electricity and Magnetism, he intended to apply to his field theory a Dynamical Philosophy and a Dynamical Approach (henceforth: DA). He affirmed that in this approach his aim was to cultivate Lagrange's dynamical theories as formulated in his Mechanique Analitique. In M.'s views, to be "dynamical”, a theory needed to provide expressions only for the kinetic and potential energies of the system, while the co-ordinates of its presumed component particles did not need directly to figure in the equations. In my study, I maintain that Maxwell's electromagnetism was compatible with Lagrange's mathematical theory of motion, because both theories take into account only the formal aspects of motion, independently from the nature of the moving particles. For the same reason, Maxwell opposed any particulate theory of the molecular state, and favored Thomson's concept of vortexes as the sole theory that explained the variety of spectroscopic lines in a gas.

Let us remark that it was through his particular approach to Lagrange's equations that M. allowed disregarding the inner mechanism of motions which generate the electromagnetic field, and he succeeded in establishing a quasi-mechanical theory of the same field. I maintain that it can be reasonably argued that the same Maxwell's DA to electromagnetic theory was at the basis of statistical approach to the kinetic theory of gases. In my view, by underlying the equal approach to the two theories, Maxwell intended to bridge the gap between the two contrasting paradigms of particle and waves.

\section{M.'s Dynamical Approach to a Field Theory of Electricity and Magnetism}

In his Dynamical Approach to a Field Theory of Electricity and Magnetism, Maxwell took advantage of Lagranges' Mechanique Analitique as a source for his equations of the electromagnetic field. It is then worth to emphasize that in his Mechanique Joseph-Luis Lagrange (1736-1812) introduced generalized co-ordinates in place of quantities representing positions, constraint-forces, velocities of the particles, the traditional parameters of motion of the Newtonian-Laplacian mechanics, eliminating these quantities from the equations. M. profited of this elimination in order "to avoid the explicit consideration of the motion of any part of the system, except the co-ordinates or variables on which the motion of the whole depends" [my Italics]. He selected appropriate expressions for the field-energy densities, choosing to represent as kinetic and potential the energies of the magnetic and electric fields respectively ${ }^{1}$.

Let us notice that in his search for an electromagnetic field, the central core of M.'s. procedure consisted in a comparison of his electromagnetic system with a special a special form of the Lagrangian in the investigation of Thomson and Tait. The interplay between the connected parts of the two systems, represented the basis for M.’s "dynamics of a connected system"2.

He considered his equations as a special case of the "equations of motion of a connected system".

His views are clearly stated in the following passage:

In this outline of the fundamental principles of the dynamics of a connected system, we have kept out of view the mechanism by which the parts of the system are connected. We have not even written down a set of equations to indicate how the motion of any part of the system depends on the variation of the variables. We have confined our attention to the variables, their velocities and momenta, and the forces which act on the pieces representing the variables. Our only assumptions are that the connections of the system are such that the time is not explicitly contained in the equations of condition, and that the principle of the conservation of energy is applicable to the system ${ }^{3}$.

The scientific and philosophical interest of M.'s original DA to his field theory is worthy of a few pages of illustration. I shall start by referring to relevant passages in Maxwell's papers. In his 1873 "On the Dynamical Evidence of the Molecular Constitution of Bodies", one of his main papers on "Molecular Science", his peculiar form of micro-physics, he turned to his preferred method, "the dynamical explanation of phenomena":

\footnotetext{
${ }^{1}$ D’Agostino (1968).

${ }^{2}$ Buchwald (1985), XII. As remarked by Buchwald, historians, physicists and philosophers used to interpret Maxwell’s method as a method mainly based on analogies, thus missing what was typical in Maxwell's DA.

${ }^{3}$ Maxwell (undated), Matter and Motion, Dover Reprint., 135. Maxwell (1954) Vol. 2, 209.
} 
When a physical phenomenon can be completely described as a change in the configuration and motion of a material system, the dynamical explanation of that phenomenon is said to be complete. We cannot conceive any further explanation to be either necessary, desirable, or possible, for as soon as we know what is meant by the words configuration, motion, mass, and force, we see that the ideas which they represent are so elementary that they cannot be explained by means of anything else $\{\text { Italics mine }\}^{4}$.

Notice that, in the above passage, Maxwell affirmed that the dynamical explanation was complete in itself, because he did not consider any further explanation of the mechanical-atomistic type to be either possible or desirable. In an essay titled “Atom”, he justified his criticism of any mechanical-atomistic explanation through the difficulty of explaining by the small and hard atomic bodies the variety of spectroscopic lines in gases: "The small hard body imagined by Lucretius and adopted by Newton, was invented for the express purpose of accounting for the permanence of the properties of bodies. But it fails to account for the vibrations of a molecule as revealed by the spectroscope" ${ }^{, 5}$. (Italics S.D.).

In fact, in the continuation of the passage, Maxwell expressed his agreement with Helmholtz' molecular theory of the vortex ring, "considered "as the true form of atom by Thomson [which] satisfies more of the conditions than any atom hitherto imagined”“ . Let us notice that M. distinguishes between an atomistic and a vortex conceptions of molecules. No doubt that he criticizes the first, and adheres to the second theory.

In his 1870 Address to the Mathematical and Physical Sections of the British Association ${ }^{7}$, after citing Thomson's theory as that one which "seeks the properties of molecules in the ring-vortices of a uniform, frictionless, incompressible fluid”, he added:

If a theory of this kind should be found, after conquering the enormous mathematical difficulties of the subject, to represent in any degree the actual properties of matter, it will stand in a very different scientific position from those theories of molecular action which are formed by investing the molecule with an arbitrary system of central forces invented expressly to account for the observed phenomena... even in the present undeveloped state of the theory, the contemplation of the individuality and indestructibility of a ring-vortex in a perfect fluid cannot fail to disturb the commonly received opinion that a molecule, in order to be permanent, must be a very hard body ${ }^{8}$. (Italics S.D.).

I argue that Maxwell's choice of Thomson's vortex theory was motivated by the same ideas expressed in his DA, as is proved by the following passage, reported from his paper “Atom”, published on the Encyclopaedia Britannica.

...The greatest recommendation of this theory \{Thomson's theory\} from a philosophical point of view, is that its success in explaining phenomena does not depend on the ingenuity with which it contrives "save appearances”, by introducing first one hypothetical force and then another. When the vortex atom is once set in motion, all its properties are absolutely fixed and determined by the laws of motion of the primitive fluid, which are fully expressed in the fundamental equations ${ }^{9}$.

The fact that the vortex atom avoided intermolecular forces was for Maxwell another point of merit for the vortex theory, one that accrued to its philosophical value.

These ideas received an outstanding confirmation in his 1877 booklet "Matter and Motion”10. Thereafter Maxwell's criticism of an atomistic approach was extended to the whole of microphysics, which he labeled as "the investigation of the mode in which the minute particles of bodies act on each other". He criticized the hypothesis that "the configuration, motion, or action of the material systems is of a certain definite kind...", because hypotheses of this type led for him to uncertain results. In Maxwell's own words: "If... the configuration, motion, or action of the material system is of a certain definite kind, and if the results of this hypothesis agree with the phenomena, then, unless we can prove that no other hypothesis would account for the phenomena, we must still admit the possibility of our hypothesis being a wrong one $\mathrm{e}^{11}$. (Italics: S.D.).

\footnotetext{
${ }^{4}$ Maxwell(1954a), Vol. 2, 418.

${ }^{5}$ Maxwell (1954) a, Vol. 2, 471.

${ }^{6}$ Maxwell (1954a), Vol. 2, 471.

${ }^{7}$ Maxwell (1954) a, 215-229, 223.

${ }^{8}$ Maxwell (1954a), Vol. 2, 223-224.

${ }^{9}$ Maxwell, “Atom”, Encyclopaedia Britannica, 445-484, 471.

${ }^{10}$ Maxwell, Matter and Motion, Dover Reprint (undated).

${ }^{11}$ Maxwell Matter and Motion, Dover Reprint (undated), 122.
} 
Maxwell's ideas had an important seguito in his master opus: A Treatise on Electricity and Magnetism ${ }^{12}$. In chapter IX, entitled: "General equations of the electromagnetic field”, he explained the meaning of a dynamical system:

In our theoretical discussion of electrodynamics we began by assuming that a system of circuits carrying electric currents is a dynamical system, in which the currents may be regarded as velocities, and in which the coordinates corresponding to these velocities do not themselves appear in the equations. It follows from this that the kinetic energy of the system, in so far as it depends on the currents, is a homogeneous quadratic function of the currents, in which the coefficients depend only on the form and relative position of the circuits. Assuming these coefficients to be known, by experiment or otherwise, we deduced, by purely dynamical reasoning, the laws of the induction of currents, and of electromagnetic attraction. In this investigation we introduced the conceptions of the electro kinetic energy of a system of currents, of the electromagnetic momentum of a circuit, and of the mutual potential of two circuits ${ }^{13}$. (Italics mine).

Leaving to the relevant bibliography comments on the technical aspects of M.'s choice of the mathematical form of the equations ${ }^{14}$, I will just remark that Maxwell's DA was not compatible with a conception of the particulate nature of electricity ${ }^{15}$, and that he could not insert in his theory a microscopic convective conception of the conduction current, i.e. the idea that a current consists in microscopic charges in motion in a wire ${ }^{16}$.

\section{Maxwell's Statistical Approach to Gas Theory as a Special Case of His DA}

In this study, I argue that Maxwell's most sophisticated treatment of his kinetic theory bears a close analogy with his Lagrangian treatment of electrodynamics. As a support to my thesis, let me mention Norton Wise's position: he complains that Maxwell's dynamical ideas have not been taken in due account by his commentators in discussing his views on gas theory ${ }^{17}$.

Concerning the role that Maxwell attributed to the relation between his statistical laws of the gases theory and traditional mechanics, let us remark that $\mathrm{M}$. highlighted a disjunction between laws of mechanics and the second law of thermodynamics. As Harman very appropriately remarks in his Introduction to Maxwell's Scientific Papers and Letters, "Maxwell's disjunction [ ]... led him to be severely critical of the attempts, notably by Clausius and Boltzmann, to reduce the second law of thermodynamics to a theorem in dynamics"18. In fact, one can clearly distinguish three different approaches to Maxwell's gas theories: one is described as purely Thermodynamics, as presented in the phenomenological Clausius' theory, to which Maxwell himself gave important contributions, such as the distribution-law of velocities and the equipartition of energy. Another approach was described as purely Dynamical, and he referred to some attempts of Clausius 1872 at a pure Dynamical theory of Thermodynamics. He quoted a few Clausius' papers as examples thereof. In his paper "Tait's Thermodynamics" he affirmed that the attempts to deduce the Second law from purely dynamical principles were unsuccessful because they led to unsound results (he quotes as an example Clausius (1872)). One may infer that his criticism was also referred to Boltzmann's attempts at a mechanical explanation of the second law. The third approach was inspired by his conception of DA, and M. labeled it as a statistical approach. The latter was for him ruled by a statistical law, which applies to systems of molecules, not the fluctuations of individual molecules. The efficaciousness of his statistical approach is opposed to Boltzmann' difficulties as a consequence of the failure of his mechanical reduction approach to gas theory. Might the weight of references in M.'s published papers lead us to accept the imposing thesis of a radical controversy that divides the two great scientists, to whom we owe fundamental steps in the kinetic gas theory? ${ }^{19}$

\footnotetext{
${ }^{12}$ Maxwell (1954).

${ }^{13}$ Maxwell (1891), (1954), Part IV, Chapt. IX, §.604, 247. According to Buchwald, the same ideas were shared by the Maxwellian, the physicists who pursued Maxwell’s approach (Buchwald (1985) 96 ff.).

${ }^{14}$ Margaret Morrison, "Unifying Scientific Theories. Physical Concepts and Mathematical Structures”, in Zeitler (2013) xxx.

${ }^{15}$ Jed Buchwald showed in detail these topics in his book: Buchwald (1985), 27-33, 37-40, 47.

${ }^{16}$ On this point: D’Agostino (1996) 5-51.

${ }^{17}$ Wise (1992), (Part 1, 191, 185,196-198). In his review paper, dealing extensively with Maxwell's dynamical ideas, Norton Wise refers to G.G. Stokes' distinction between "mechanical” and “dynamical” theories, the latter consisting in a methods that does not require the physicist "to assume either that the ether does or that it does not, consist of distinct particles" (Wise [1992] 186).

${ }^{18}$ Harmann (1995), 171-213.

${ }^{19}$ As regards my interpretation of Maxwell's famous demon, I believe that his discredit of atomism can be reasonably transferred to M.'s view of his metaphor as an absurd falsification of the second termodynamic second law (Harmann [1995] 18)], a metaphorical example of the fact that the demonic account of gas particles, led to the paradox of denying the thermodynamic second law.
} 
In this study, I support the thesis: Maxwell's statistical approach to his Gas Theory was consistent with his DA to his electromagnetic theory, and with his accepted vortex molecular approach. In fact, in an Appendix Maxwell added on May 1879 to one of his last essays on kinetic theory, he characterized his statistical approach in the following passage:

The method, which I have employed throughout, is a purely statistical one. It considers the mean values of certain functions of the velocities within a given element of the medium, but it never attempts to trace the motion of a molecule, not even as far as to estimate the length of its mean path. Hence all the equations are expressed in the form of a differential calculus, in which the phenomena at a certain place are connected with the space variations of certain quantities at that place, but in which no quantity appears which explicitly involves the conditions of things at a finite distance from that place ${ }^{20}$ (Italics: S.D.).

In my italicized lines, I wish to call attention to Maxwell's accuracy in excluding to introduce mechanical features in the molecular motions, not even the mean path length of molecular percorses, and in his emphasis on his locally continuous approach to molecular space. He adds that in this work he adopted the same statistical method of his 1867 fundamental kinetic paper: Dynamical Theory of Gases ${ }^{21}$.

In spite of his assertion to disregard hypotheses on particulate molecules, Maxwell's DA did not consist however in abandoning hypotheses altogether, but in framing only theories related to "the most general properties of material systems". His rejection of hypotheses on atoms and molecules as "small hard bodies" was consistent with the necessity of framing only hypotheses of the most general type such as those implicit in his DA. In a synthesis, he cast a judgment of fundamental ambiguity on hypotheses about the configuration, motion, or action of the material system, i.e., hypotheses of a certain definite type, because he was convinced that their experimental proof was not crucial. Among the most general hypotheses, one was for him extremely general: that on a state of motion independent from the nature of the moving particles:

The extremely general \{hypothesis\}... is ...that the phenomena to be investigated depend on the configuration and motion of a material system... If our hypothesis is the extremely general one that the phenomena to be investigated depend on the configuration and motion of a material system, then if we are able to deduce any available result from such an hypothesis, we may safely apply them to the phenomena before us ${ }^{22}$.

That phenomena to be investigated depend on the configuration and motion of a material system, his DA, is here considered as the sole easily experimentally testable hypotheses. Clearly, Maxwell's position prefigures an ante-literam Popperian view of falsificationism. But at difference with Karl Popper's view, M.' criterium is founded on the role and nature of theories, not to the falsification of experiments.

\section{Conclusive Remarks}

In accordance with my study above on Maxwell's Dynamical Approaches to a field theory of Electromagnetism and to a Gas Kinetic theory, I believe that I may consistently support the point that by this DA Maxwell intended to bridge the gap between fields and particles, the two fundamentally contrasting paradigms of the Middle Nineteenth Century physics. His bridging implied establishing an analogy between his electromagnetic system of fields and currents and his interpretation of the Lagrangian equations. To the opposite view that this analogy was inconsistent, because Maxwell's bridging procedure amounted to no less than an attempt to solve the contrast between electromagnetism and mechanics ${ }^{23}$, I oppose my account of the very features of M.'s DA: the feature of Lagrange's theory as a form of a mathematical theory that had lost any connection with a particulate mechanics, and the DA feature of M.' as a non particulate despite mechanical approach to his electromagnetic system.

M.'s great genius was expressed in his original interpretation of Lagrange's Mechanique, and in his original comparison of his electromagnetic system of fields and currents with the above specially connected Lagrangian system. They were both necessary and sufficient conditions to bridge the paradigmatic gap between particles and fields.

\footnotetext{
${ }^{20}$ Maxwell, Appendix to “On Stresses in Rarefied Gases arising from Inequalities in Temperature”, added on May 1879 in: Maxwell (1954a) Vol. 2, 703-712, 704.

${ }^{21}$ Maxwell (1954a) Vol. 2, 681-712, 681.

${ }^{22}$ Maxwell (undated) Dover Publ., 122.

${ }^{23}$ [Neressian (Meheus, 2002)].
} 
It is true, and can be easily accepted that, after all, M.'s DA to his Field and Kinetic theories, presented in the form of quasi-mechanical theories, were in "the spirit of the dynamic tradition". According to Rosenfeld's views, however, after Maxwell "the spirit of the dynamic tradition which had achieved so much was on the wane. The discovery of the electron and the study of fast electrified particles raided more pressing practical problems, ... [and] the problem of the structure of the ether appeared quasi superfluous: the electromagnetic concepts and equations formed a self-contained, consistent system, offering a firm starting point for the analysis of the new phenomena" 24 . The electromagnetic field "had now become as concrete a reality as any dynamical system and could serve as a complete substitute for the ether". The whole situation conspired for encouraging a new phenomenological conception of theory, and M.’s imposing electromagnetic system appeared to Heaviside, Lorentz, and their successors an altogether useless effort. It simply decayed from these physicists' interests.

As is known, Maxwell's theory, achieved enormous successes and we are still immersed in the theoretical and technological developments that his theory made possible. The Maxwellian synthesis therefore cannot appear to us as a useless achievement.

But the historian cannot fail to remark that these successes were the fruit of mutations in the theoretical and experimental approaches to Nineteenth physics. If it is true that a higher aspect of continuity is to be admitted, and that the mathematics of M.' equations represented a form of continuity above the change of physical concepts, and of M.'s intrinsic Philosophy, it is also to be remarked that our equations differ from M.'s original vector potential equations, for many aspects, and primarily because of Heaviside and Hertz's vectorial simplification. Our theories are not the issue of a continuous line of development of the foundational conceptions that supported Maxwell's theory. But, right because of this view, if we look today from our modern positions at M.'s DA, and to his quasi-mechanical field theories, his enterprise appears to us as a fantastic contribution to the so called "Logic of Discovery", and we bow with reverence when recalling what he achieved with them, and what he left to Einstein and his successors. A new solution of the mechanic-field problem in the form of a revolutionary Philosophy and of new approach to physics, arrived indeed after fifty years from an unexpected side, Albert Einstein's Special Relativity and his generalized field theories.

\section{References}

Buchwald, Z. J. (1985). From Maxwell to Microphysics, Aspects of the Electromagnetic Theory in the Last Quarter of the Nineteenth Century. Chicago: The University of Chicago Press.

Clausius, R. (1872). Uber die verschiedenen Maassysteme... Annalen der Physik und Chemie. N.F. Band XVI.

D’Agostino, S. (1996). Absolute Systems of Unites and Dimensions of Physical Quantities: A Link between Weber's Electrodynamics and Maxwell's Electromagnetic Theory of Light. Physis, XXXIII, Nuova Serie, Fasc.1-3, 5-51.

Harmann, P. M. (Ed.) (1995). The Scientific Papers and Letters of James Clerk Maxwell. Cambridge University Press, 2, 1862-1873.

Maxwell, J. C. (1855). On Faraday’s Lines of Force. Maxwell (1954), Vol. 1, 155-229.

Maxwell, J. C. (1861). On Physical Lines of Force. Philosophical Magazine, 21; Part I, March Ibid, Part II, April and May 1861.

Maxwell, J. C. (1877). Matter and Motion 1901-1921. Amsterdam: Elsevier.

Maxwell, J. C. (1890). The Scientific Papers of James Clark Maxwell (3rd ed.). Vol. 2.

Maxwell, J. C. (1891). A Treatise on Electricity and Magnetism (3rd ed.). Cambridge: Cambridge University Press.

Maxwell, J. C. (1954). A Treatise on Electricity and Magnetism. Vol. 2, Dover Reprint.

Maxwell, J. C. (1954a). The Scientific Papers of James Clark Maxwell. Vol. 2, Dover Reprint.

Maxwell, J. C. (1995). Scientific Papers and Letters. Vol. 2, 1862-1873, Cambridge: Cambridge University Press.

Maxwell, J. C. (Circa) (1871). On the Mathematical Classification of Physical Quantities. (Undated) Ibid, Maxwell (1954), Vol. 2, 257-266.

Maxwell, J. C. (Circa) (1920). Matter and Motion. Reprinted, with Notes and an Appendix by Sir Joseph Larmor.

Maxwell, J. C. (Undated). Matter and Motion. Dover Reprint.

Meheus (Ed.) (2002). Inconsistencies in Science. Dordrecht: Kluwer Academics Publishers.

\footnotetext{
${ }^{24}$ [Rosenfeld (1956)]. According to Rosenfeld, M.’s Philosophy was also superseded by Hertz's experimental production of Maxwell's electromagnetic waves.
} 
Rosenfeld, L. (1956). The Velocity of Light and the Evolution of Electrodynamics. Il Nuovo Cimento, Supplemento al Vol. IV, s. X, 5, 1630-1667.

Wise, N. (1992). The Maxwell Literature and British Dynamical Theory. Historical Studies in the Physical Sciences, Vol. 13, Part 1, 175-206.

Zeitler, P. (2013). Models and Metaphors as Revolutionary Tools in Science. Berlin: LT Verlag. 